\title{
Measure of weak noncompactness under complex interpolation
}

\author{
by \\ Andrzej Kryczka and StanisŁaw Prus (Lublin)
}

\begin{abstract}
Logarithmic convexity of a measure of weak noncompactness for bounded linear operators under Calderón's complex interpolation is proved. This is a quantitative version for weakly noncompact operators of the following: if $T: A_{0} \rightarrow B_{0}$ or $T: A_{1} \rightarrow B_{1}$ is weakly compact, then so is $T: A_{[\theta]} \rightarrow B_{[\theta]}$ for all $0<\theta<1$, where $A_{[\theta]}$ and $B_{[\theta]}$ are interpolation spaces with respect to the pairs $\left(A_{0}, A_{1}\right)$ and $\left(B_{0}, B_{1}\right)$. Some formulae for this measure and relations to other quantities measuring weak noncompactness are established.
\end{abstract}

1. Introduction. Measures of noncompactness or weak noncompactness have been successfully applied in operator theory, differential equations, and integral equations (see [1], [5], and [6]). In particular, they enabled several authors to develop quantitative methods in interpolation of operators (see [2], [11]-[15], [28]). One of the main aims of these investigations is to establish a kind of logarithmic convexity of the measures under interpolation. Most results in this direction were obtained for real interpolation. Some of them generalize the following Beauzamy theorem [9]: if the embedding $I: A_{0} \cap A_{1} \rightarrow A_{0}+A_{1}$ is weakly compact then the interpolation spaces $A_{\theta, p}$ are reflexive for all $0<\theta<1$ and $1<p<\infty$. In this paper we consider Calderón's complex interpolation. The counterpart of Beauzamy's result is false for this interpolation method (see [25]). Nevertheless, Calderón [10] proved that if one of the Banach spaces $A_{0}, A_{1}$ is reflexive then so is the interpolation space $A_{[\theta]}$ for every $0<\theta<1$.

In [24], a measure of weak noncompactness $\gamma$ for sets and a corresponding measure $\Gamma$ for operators were introduced. The measure $\Gamma$ was applied to the Lions-Peetre real interpolation method (in a discrete form). Namely, for all $0<\theta<1$ and $1<p<\infty$ the following estimate was established:

$$
\Gamma_{\theta, p}(T) \leq c_{\theta} \Gamma_{0}(T)^{1-\theta} \Gamma_{1}(T)^{\theta},
$$

where $c_{\theta}=2^{\theta(1-\theta)}$ and $\Gamma_{\theta, p}(T), \Gamma_{j}(T), j=0,1$, are values of $\Gamma$ for the

2000 Mathematics Subject Classification: 46B70, 46M35. 
operators $T: A_{\theta, p} \rightarrow B_{\theta, p}$ and $T: A_{j} \rightarrow B_{j}, j=0,1$ respectively. In this paper we obtain an analogous estimate for complex interpolation, but with $c_{\theta}=1$. This generalizes Calderón's above-mentioned result. We also show that $\Gamma$ is equivalent to a quantity measuring the deviation from weak compactness of an operator, which was studied by González, Saksman and Tylli in [21]. Consequently, an estimate of type (1.1) holds for this quantity as well.

Our notation and terminology are standard. Given a Banach space $X$ the open unit ball of $X$ will be denoted by $\mathrm{B}_{X}$ and its closure by $\overline{\mathrm{B}}_{X}$. The family of all nonempty bounded subsets of $X$ will be denoted by $\mathcal{M}_{X}$. We write conv $A$ for the convex hull of a set $A \subset X$ and Conv $A$ for the closure of conv $A$. Throughout this paper elements of $X$ are identified with their canonical images in the second dual $X^{* *}$. Abbreviations and symbols beginning with $w^{*}$ refer to the weak-star topology. The space of all bounded linear operators between Banach spaces $X$ and $Y$ will be denoted by $L(X, Y)$.

2. Measures of weak noncompactness. For simplicity, in the first three sections of this paper we restrict our attention to real Banach spaces. However, all proofs can be easily extended to complex Banach spaces, so the results can be applied in the last section where we consider complex spaces.

Let $X$ be a Banach space and let $\left(x_{n}\right)$ be a sequence in $X$. We say that $\left(y_{n}\right)$ is a sequence of successive convex combinations (scc for short) for $\left(x_{n}\right)$ if there exists a sequence of integers $0=p_{1}<p_{2}<\ldots$ such that $y_{n} \in \operatorname{conv}\left\{x_{i}\right\}_{i=p_{n}+1}^{p_{n+1}}$ for each $n$. Of course, the relation scc is reflexive and transitive. Similarly, vectors $u_{1}, u_{2}$ are said to be a pair of scc for $\left(x_{n}\right)$ if $u_{1} \in \operatorname{conv}\left\{x_{i}\right\}_{i=1}^{p}$ and $u_{2} \in \operatorname{conv}\left\{x_{i}\right\}_{i=p+1}^{\infty}$ for some integer $p \geq 1$. From our point of view, the crucial fact about sequences of scc is the following theorem based on an idea of Milman [26]. For the convenience of the reader we repeat the proofs of the next two theorems from [24].

TheOREM 2.1. Let $\left(x_{n}\right)$ be a bounded sequence in a Banach space X. For every $\varepsilon>0$ there exists a sequence $\left(y_{n}\right)$ of scc for $\left(x_{n}\right)$ such that if $u_{1}, u_{2}$ and $v_{1}, v_{2}$ are any pairs of scc for $\left(y_{n}\right)$, then $\left|\left\|u_{1}-u_{2}\right\|-\left\|v_{1}-v_{2}\right\|\right| \leq \varepsilon$.

Proof. Let $A_{n}=\operatorname{Conv}\left\{x_{i}\right\}_{i=n}^{\infty}$ for $n \in \mathbb{N}$. Assume first that $\bigcap_{n=1}^{\infty} A_{n} \neq \emptyset$. Then we can find a convergent sequence of scc for $\left(x_{n}\right)$ and, by ignoring a finite number of terms if necessary, we get a sequence as required. Suppose now that $\bigcap_{n=1}^{\infty} A_{n}=\emptyset$. A reasoning similar to that in the proof of the corollary of Theorem 2 in [26] (see also [27]) gives a constant $d>0$ such that for any $\varepsilon>0$ there exists a sequence $\left(y_{n}\right)$ of scc for $\left(x_{n}\right)$ such that $d-\varepsilon \leq\left\|u_{1}-u_{2}\right\| \leq d$ for any pair $u_{1}, u_{2}$ of scc for $\left(y_{n}\right)$. Clearly, $\left(y_{n}\right)$ fulfills the assertion of the theorem. 
The notion of scc was used in [24] to define a measure of weak noncompactness $\gamma$ which is a counterpart for the weak topology of the separation measure of noncompactness (see [1], [5]). By the convex separation of $\left(x_{n}\right)$ we mean

$$
\operatorname{csep}\left(x_{n}\right)=\inf \left\{\left\|u_{1}-u_{2}\right\|: u_{1}, u_{2} \text { is a pair of scc for }\left(x_{n}\right)\right\} .
$$

For each $A \in \mathcal{M}_{X}$ we put

$$
\gamma(A)=\sup \left\{\operatorname{csep}\left(x_{n}\right):\left(x_{n}\right) \subset \operatorname{conv} A\right\} .
$$

The function $\gamma$ is a measure of weak noncompactness in the sense of the axiomatic definition given in [7]. Namely, for all sets $A, B \in \mathcal{M}_{X}$ and scalars $\lambda$ we have

(1) $\gamma(A)=0$ if and only if $A$ is a relatively weakly compact set;

(2) $\gamma(\operatorname{Conv} A)=\gamma(A)$;

(3) $\gamma(A \cup B)=\max \{\gamma(A), \gamma(B)\}$;

(4) $\gamma(A+B) \leq \gamma(A)+\gamma(B)$;

(5) $\gamma(\lambda A)=|\lambda| \gamma(A)$

(see [24]). Condition (1) is a consequence of James' criterion of weak compactness (see [22]). Using an idea from [22] one can also obtain the following formulae [24].

Theorem 2.2. Let $X$ be a Banach space and $A \in \mathcal{M}_{X}$. Then

$$
\begin{aligned}
\gamma(A)= & \sup \left\{\lim _{n} \lim _{k} F_{n}\left(x_{k}\right)-\lim _{k} \lim _{n} F_{n}\left(x_{k}\right):\right. \\
& \left.\left(x_{k}\right) \subset \operatorname{conv} A,\left(F_{n}\right) \subset \overline{\mathrm{B}}_{X^{*}} \text { and the limits exist }\right\} \\
= & \sup \operatorname{dist}\left(x^{* *}, \operatorname{conv}\left\{x_{n}\right\}\right),
\end{aligned}
$$

where the second supremum is taken over all sequences $\left(x_{n}\right)$ in $\operatorname{conv} A$ and all $w^{*}$-cluster points $x^{* *} \in X^{* *}$ of $\left(x_{n}\right)$.

Proof. We first prove (2.2). Denote by $\gamma^{\prime}(A)$ the right-hand side of $(2.2)$. In order to show that $\gamma^{\prime}(A) \geq \gamma(A)$ we argue as in the proof of Lemma 2.1 of [27]. Indeed, for $\varepsilon>0$ take a sequence $\left(x_{n}\right)$ in conv $A$ such that $\gamma(A)-$ $\varepsilon \leq \operatorname{csep}\left(x_{n}\right)$ and fix $x \in \operatorname{conv}\left\{x_{n}\right\}$. There exists $m \in \mathbb{N}$ such that if $y \in$ $\operatorname{conv}\left\{x_{i}\right\}_{i=m}^{\infty}$, then $x, y$ is a pair of scc for $\left(x_{n}\right)$ and therefore $\|x-y\| \geq$ $\operatorname{csep}\left(x_{n}\right) \geq \gamma(A)-\varepsilon$. By applying a separation theorem, we can find a functional $x^{*} \in X^{*}$ such that $\left\|x^{*}\right\| \leq 1$ and $x^{*}(y-x) \geq \gamma(A)-\varepsilon$ for all $y \in \operatorname{conv}\left\{x_{i}\right\}_{i=m}^{\infty}$. Let $x^{* *} \in X^{* *}$ be a $w^{*}$-cluster point of $\left(x_{n}\right)$. Then $x^{* *}\left(x^{*}\right)=\lim _{k \rightarrow \infty} x^{*}\left(x_{n_{k}}\right)$ for some subsequence $\left(x_{n_{k}}\right)$ and consequently $\left\|x^{* *}-x\right\| \geq\left(x^{* *}-x\right)\left(x^{*}\right)=\lim _{k \rightarrow \infty} x^{*}\left(x_{n_{k}}-x\right) \geq \gamma(A)-\varepsilon$. This gives $\operatorname{dist}\left(x^{* *}, \operatorname{conv}\left\{x_{n}\right\}\right) \geq \gamma(A)-\varepsilon$ and finally $\gamma^{\prime}(A) \geq \gamma(A)$.

The proof of the opposite inequality is a modification of a reasoning in [22] (see also [18]). For each $\varepsilon>0$ there exists a sequence $\left(x_{n}\right)$ in conv $A$ 
and its $w^{*}$-cluster point $x^{* *} \in X^{* *} \operatorname{such}$ that $\operatorname{dist}\left(x^{* *}, \operatorname{conv}\left\{x_{n}\right\}\right) \geq \gamma^{\prime}(A)-\varepsilon$. By a separation theorem we obtain a functional $x^{* * *} \in X^{* * *}$ such that $\left\|x^{* * *}\right\| \leq 1$ and $x^{* * *}\left(x^{* *}-x\right) \geq \gamma^{\prime}(A)-\varepsilon$ for all $x \in \operatorname{conv}\left\{x_{n}\right\}$. We now choose by induction a sequence of functionals $\left(x_{k}^{*}\right) \subset X^{*}$ and a subsequence $\left(x_{n_{k}}\right)$ with $\left\|x_{k}^{*}\right\| \leq 1+\varepsilon$ for all $k \in \mathbb{N}, x_{k}^{*}\left(x_{n_{i}}\right) \geq x^{* * *}\left(x^{* *}\right)-\varepsilon$ if $k \leq i$ and $x_{k}^{*}\left(x_{n_{i}}\right)=x^{* * *}\left(x_{n_{i}}\right)$ if $i<k$. By the principle of local reflexivity [18, p. 33], there exists $x_{1}^{*} \in X^{*}$ such that $\left\|x_{1}^{*}\right\| \leq 1+\varepsilon$ and $x^{* *}\left(x_{1}^{*}\right)=x^{* * *}\left(x^{* *}\right)$. Since $x^{* *}$ is a $w^{*}$-cluster point of $\left(x_{n}\right)$ we can find $n_{1} \in \mathbb{N}$ satisfying $\left|x_{1}^{*}\left(x_{n_{1}}\right)-x^{* *}\left(x_{1}^{*}\right)\right| \leq \varepsilon$.

Assume now that we have obtained the required $x_{1}^{*}, \ldots, x_{k-1}^{*}$ and $x_{n_{1}}, \ldots, x_{n_{k-1}}$. There exists $x_{k}^{*} \in X^{*}$ such that $\left\|x_{k}^{*}\right\| \leq 1+\varepsilon, x_{k}^{*}\left(x_{n_{i}}\right)=$ $x^{* * *}\left(x_{n_{i}}\right)$ for $i=1, \ldots, k-1$ and $x^{* *}\left(x_{k}^{*}\right)=x^{* * *}\left(x^{* *}\right)$. Choose $n_{k}>n_{k-1}$ satisfying $\left|x_{i}^{*}\left(x_{n_{k}}\right)-x^{* *}\left(x_{i}^{*}\right)\right| \leq \varepsilon$ for $i=1, \ldots, k$. Consider now a pair $u, v$ of scc for $\left(x_{n_{i}}\right)$. There exists $k>1$ such that $u \in \operatorname{conv}\left\{x_{n_{i}}\right\}_{i=1}^{k-1}$ and $v \in \operatorname{conv}\left\{x_{n_{i}}\right\}_{i=k}^{\infty}$. Of course $x_{k}^{*}(u)=x^{* * *}(u)$ and $x_{k}^{*}(v) \geq x^{* * *}\left(x^{* *}\right)-\varepsilon$, and hence $(1+\varepsilon)\|u-v\| \geq x_{k}^{*}(v-u) \geq x^{* * *}\left(x^{* *}-u\right)-\varepsilon \geq \gamma^{\prime}(A)-2 \varepsilon$. It follows that

$$
\operatorname{csep}\left(x_{n_{i}}\right) \geq \frac{\gamma^{\prime}(A)-2 \varepsilon}{1+\varepsilon},
$$

which gives

$$
\gamma(A) \geq \frac{\gamma^{\prime}(A)-2 \varepsilon}{1+\varepsilon} .
$$

Letting $\varepsilon \rightarrow 0$ we obtain $\gamma(A) \geq \gamma^{\prime}(A)$, and the proof of (2.2) is complete.

Denote by $e(A)$ the right-hand side of (2.1). From the proof of (2.2) it follows that there exists $a$ such that for every $\varepsilon>0$ one can find sequences $\left(x_{k}\right) \subset \operatorname{conv} A$ and $\left(F_{n}\right) \subset \overline{\mathrm{B}}_{X^{*}}$ with $F_{n}\left(x_{k}\right) \geq a-\varepsilon$ for $n \leq k$ and $F_{n}\left(x_{k}\right) \leq$ $a-\gamma(A)+\varepsilon$ for $n>k$. By passing to a subsequence if necessary, we can assume that the limits $\alpha_{1}=\lim _{n} \lim _{k} F_{n}\left(x_{k}\right)$ and $\alpha_{2}=\lim _{k} \lim _{n} F_{n}\left(x_{k}\right)$ both exist. Of course, $\gamma(A)-2 \varepsilon \leq \alpha_{1}-\alpha_{2}$. Hence $\gamma(A)-2 \varepsilon \leq e(A)$ and $\gamma(A) \leq e(A)$.

Let $\left(x_{k}\right) \subset \operatorname{conv} A$ and $\left(F_{n}\right) \subset \overline{\mathrm{B}}_{X^{*}}$ be any sequences for which the limits $\beta_{1}=\lim _{n} \lim _{k} F_{n}\left(x_{k}\right)$ and $\beta_{2}=\lim _{k} \lim _{n} F_{n}\left(x_{k}\right)$ exist. By Theorem 2.1 for every $\varepsilon>0$ we can find a sequence $\left(z_{k}\right)$ of scc for $\left(x_{k}\right)$ such that $\left\|z_{i}-z_{j}\right\|-\operatorname{csep}\left(z_{k}\right) \leq \varepsilon$ for every $i \neq j$. Then $\beta_{1}=\lim _{n} \lim _{k} F_{n}\left(z_{k}\right)$ and $\beta_{2}=\lim _{k} \lim _{n} F_{n}\left(z_{k}\right)$. Therefore $\beta_{1}-\beta_{2} \leq \liminf _{j} \liminf _{i}\left\|z_{i}-z_{j}\right\|$. Consequently, $\beta_{1}-\beta_{2} \leq \operatorname{csep}\left(z_{k}\right)+\varepsilon \leq \gamma(A)+\varepsilon$. Hence $\gamma(A)+\varepsilon \geq e(A)$ and finally $\gamma(A) \geq e(A)$.

Formula (2.2) suggests another way of measuring weak noncompactness.

Definition 2.3. For each $A \in \mathcal{M}_{X}$ let

$$
\bar{\gamma}(A)=\sup \operatorname{dist}\left(x^{* *}, X\right)
$$


where the supremum is taken over all $w^{*}$-cluster points $x^{* *} \in X^{* *}$ of sequences in conv $A$.

It is easy to check that $\bar{\gamma}$ is a measure of weak noncompactness. We shall show that $\bar{\gamma}$ is equivalent to $\gamma$.

Theorem 2.4. Let $X$ be a Banach space and $A \in \mathcal{M}_{X}$. Then

$$
\bar{\gamma}(A) \leq \gamma(A) \leq 2 \bar{\gamma}(A)
$$

Proof. Of course, $\bar{\gamma}(A) \leq \gamma(A)$. Fix $\varepsilon>0$. There exist a sequence $\left(x_{n}\right) \subset$ conv $A$ and its $w^{*}$-cluster point $x^{* *}$ such that $\gamma(A)-\varepsilon \leq \operatorname{dist}\left(x^{* *}, \operatorname{conv}\left\{x_{n}\right\}\right)$ $=\delta$. We write $\delta_{0}=\operatorname{dist}\left(x^{* *}, X\right)$. There is $x \in X$ such that $\left\|x^{* *}-x\right\|<\delta_{0}+\varepsilon$. Taking $\delta_{1}=\operatorname{dist}\left(x, \operatorname{conv}\left\{x_{n}\right\}\right)$ we choose $x^{*} \in \overline{\mathrm{B}}_{X^{*}}$ so that $x^{*}(x-y) \geq \delta_{1}$ for every $y \in \operatorname{conv}\left\{x_{n}\right\}$. Then $\delta_{1} \leq x^{*}(x)-x^{* *}\left(x^{*}\right) \leq\left\|x-x^{* *}\right\|<\delta_{0}+\varepsilon$. Since $\delta \leq \delta_{1}+\left\|x^{* *}-x\right\|<2\left(\delta_{0}+\varepsilon\right)$, we conclude that $\gamma(A)<2 \delta_{0}+3 \varepsilon \leq 2 \bar{\gamma}(A)+3 \varepsilon$. Finally, $\gamma(A) \leq 2 \bar{\gamma}(A)$.

The measures $\gamma$ and $\bar{\gamma}$ are equal in the space $c_{0}$ (see [24]), but in general inequalities (2.3) cannot be improved. Indeed, consider $c_{0}$ as a subspace of the space $c$. Then $\gamma\left(\bar{B}_{c_{0}}\right)=\bar{\gamma}\left(\bar{B}_{c_{0}}\right)=1$ (see [24]). On the other hand, considering elements of type $(1, \ldots, 1,-1, \ldots)$ one can see that $2=\gamma\left(\bar{B}_{c}\right)=$ $2 \bar{\gamma}\left(\overline{\mathrm{B}}_{c}\right)$.

Another example of a measure of weak noncompactness was introduced by De Blasi [16]. It is given by the formula

$$
\omega(A)=\inf \left\{t>0: A \subset C+t \overline{\mathrm{B}}_{X}, C \subset X \text { is weakly compact }\right\}
$$

for each $A \in \mathcal{M}_{X}$. This measure can be regarded as a counterpart for the weak topology of the classical Hausdorff measure of noncompactness. We have

$$
\xi(A) \leq \xi\left(\mathrm{B}_{X}\right) \omega(A)
$$

for any measure of weak noncompactness $\xi$ and every $A \in \mathcal{M}_{X}$ (see [8]).

From Theorem 2.2 and a result in [4] we see that $\gamma$ is not equivalent to $\omega$. On the other hand, these measures coincide in the space $c_{0}$ (see [24]). We shall establish relations between $\gamma, \bar{\gamma}$ and $\omega$ in the Lebesgue space $L^{1}(\mu)$. This space is especially important for the applications of measures of weak noncompactness in differential and integral equations (see [6] and the references given there). Let $\mu$ be a finite measure. Then

$$
\omega(A)=\inf _{\delta>0} \sup _{\mu(D) \leq \delta} \sup _{x \in A} \int_{D}|x(t)| d \mu(t)
$$

for every $A \in \mathcal{M}_{L^{1}(\mu)}$ (see [3]).

THEOREM 2.5. Let $\mu$ be a finite measure and $A \in \mathcal{M}_{L^{1}(\mu)}$. Then $\gamma(A)=$ $2 \omega(A)$ and $\bar{\gamma}(A)=\omega(A)$. 
Proof. From (2.4) we see that $\gamma(A) \leq 2 \omega(A)$ and $\bar{\gamma}(A) \leq \omega(A)$ for each $A \in \mathcal{M}_{L^{1}(\mu)}$. It is therefore enough to prove the opposite inequalities. We take $A \in \mathcal{M}_{L^{1}(\mu)}$ with $\omega(A)>0$ and a number $\eta$ with $0<2 \eta<\omega(A)$. Formula (2.5) shows that for every $n$ there exist a measurable set $D_{n}$ with $\mu\left(D_{n}\right) \leq 1 / 2^{n}$ and $x_{n} \in A$ such that

$$
\int_{D_{n}}\left|x_{n}(t)\right| d \mu(t)>\omega(A)-\eta
$$

We set $n_{1}=1$ and choose $n_{2}>n_{1}$ so that if $\mu(E) \leq 1 / 2^{n_{2}-1}$ then

$$
\int_{E}\left|x_{n_{1}}(t)\right| d \mu(t)<\eta \text {. }
$$

Let $F=\bigcup_{k \geq n_{2}} D_{k}$ and $E_{1}=D_{n_{1}} \backslash F$. Clearly, $\mu(F) \leq \sum_{k \geq n_{2}} 1 / 2^{k}=$ $1 / 2^{n_{2}-1}$. Hence

$$
\begin{aligned}
\omega(A)-\eta & <\int_{D_{n_{1}}}\left|x_{n_{1}}(t)\right| d \mu(t) \leq \int_{E_{1}}\left|x_{n_{1}}(t)\right| d \mu(t)+\int_{F}\left|x_{n_{1}}(t)\right| d \mu(t) \\
& <\int_{E_{1}}\left|x_{n_{1}}(t)\right| d \mu(t)+\eta
\end{aligned}
$$

and thus $\int_{E_{1}}\left|x_{n_{1}}(t)\right| d \mu(t)>\omega(A)-2 \eta$. Proceeding in this way we obtain a subsequence $\left(x_{n_{k}}\right)$ and a sequence $\left(E_{k}\right)$ of pairwise disjoint sets such that

$$
\int_{E_{k}}\left|x_{n_{k}}(t)\right| d \mu(t)>\omega(A)-2 \eta
$$

for every $k$. In what follows, $\chi_{G}$ will denote the characteristic function of the set $G$. We put $E=\bigcup_{k=1}^{\infty} E_{k}$ and $y_{k}=x_{n_{k}} \chi_{E}$ for $k=1,2, \ldots$ Following the reasoning in $[17$, p. 93] (see also [23]) we can assume that

$$
\int_{E \backslash E_{k}}\left|x_{n_{k}}(t)\right| d \mu(t)<\eta
$$

for all $k$ and then

$$
\left\|\sum_{k=1}^{n} \alpha_{k} x_{n_{k}}\right\| \geq\left\|\sum_{k=1}^{n} \alpha_{k} y_{k}\right\|>(\omega(A)-3 \eta) \sum_{k=1}^{n}\left|\alpha_{k}\right|
$$

for all scalars $\alpha_{1}, \ldots, \alpha_{n}$. This shows in particular that $\gamma(A) \geq \operatorname{csep}\left(x_{n_{k}}\right)>$ $2(\omega(A)-3 \eta)$. Consequently, $\gamma(A) \geq 2 \omega(A)$, which completes the proof of the first formula.

Now consider the functions $z_{k}=x_{n_{k}} \chi_{E_{k}}$. From (2.6) and (2.7) it follows that $\left\|z_{k}\right\|>\omega(A)-2 \eta$ and $\left\|z_{k}-x_{n_{k}}\right\|<\eta$ for every $k$. Let $Z$ be the closed linear subspace spanned by the vectors $z_{k}$. Since the sets $E_{k}$ are pairwise disjoint, there exists a linear isometry $T: Z \rightarrow l^{1}$ such that $T\left(z_{k} /\left\|z_{k}\right\|\right)$ is the $k$ th vector of the standard basis of $l^{1}$. Moreover, $Z$ is complemented in 
$L^{1}(\mu)$ by means of a norm-one projection $P$. Given $x \in L^{1}(\mu)$ we find $m$ so that $\|T z-T(P x)\| \geq\|T z\|-\eta$ for each $z$ in the linear span of $\left\{z_{k}\right\}_{k \geq m}$. Let $u$ belong to $K_{m}=\operatorname{conv}\left\{x_{n_{k}}\right\}_{k \geq m}$, i.e. $u=\sum_{k=m}^{s} \lambda_{k} x_{n_{k}}$ where $s>m$, $\lambda_{m}, \ldots, \lambda_{s} \geq 0$ and $\sum_{k=m}^{s} \lambda_{k}=1$. We set $z=\sum_{k=m}^{s} \lambda_{k} z_{k}$. Then $\|z-u\|<\eta$ and

$$
\|x-z\| \geq\|P x-z\| \geq\|T z\|-\eta=\sum_{k=m}^{s} \lambda_{k}\left\|z_{k}\right\|-\eta>\omega(A)-3 \eta,
$$

which gives $\|x-u\|>\omega(A)-4 \eta$. Consequently, $\operatorname{dist}\left(x, K_{m}\right) \geq \omega(A)-4 \eta$ for each $m$. We can now apply a reasoning similar to that in the proof of Lemma 2.1 in [26]. It shows that $\left\|x^{* *}-x\right\| \geq \omega(A)-4 \eta$ for every $w^{*}$-cluster point $x^{* *} \in X^{* *}$ of $\left(x_{n_{k}}\right)$. It follows that $\bar{\gamma}(A) \geq \operatorname{dist}\left(x^{* *}, X\right) \geq \omega(A)-4 \eta$. This finally yields $\bar{\gamma}(A) \geq \omega(A)$.

3. Weak noncompactness of operators. Let $X$ and $Y$ be Banach spaces and $T \in L(X, Y)$. We set $\Gamma(T)=\gamma\left(T\left(\mathrm{~B}_{X}\right)\right)$ and $\bar{\Gamma}(T)=\bar{\gamma}\left(T\left(\mathrm{~B}_{X}\right)\right)$. Clearly, each of the conditions $\Gamma(T)=0, \bar{\Gamma}(T)=0$ is equivalent to weak compactness of $T$. The functions $\Gamma$ and $\bar{\Gamma}$ will therefore be called measures of weak noncompactness for operators. They are also seminorms in $L(X, Y)$. A different way of measuring weak noncompactness of operators was discussed in [21] (see also [29]). Namely, to each $T \in L(X, Y)$ there corresponds the operator $R(T): X^{* *} / X \rightarrow Y^{* *} / Y$ given by the formula

$$
R(T)\left(x^{* *}+X\right)=T^{* *} x^{* *}+Y
$$

for every $x^{* *} \in X^{* *}$. Then

$$
\|R(T)\|=\sup \left\{\operatorname{dist}\left(T^{* *} x^{* *}, Y\right): \operatorname{dist}\left(x^{* *}, X\right) \leq 1\right\},
$$

and $\|R(T)\|=0$ if and only if $T$ is weakly compact (see [19, p. 482]). We shall prove that $\Gamma, \bar{\Gamma}$ and $\|R(\cdot)\|$ are equivalent.

Lemma 3.1. Let $X$ and $Y$ be Banach spaces and $T \in L(X, Y)$. Then

$$
\|R(T)\|=\sup \left\{\operatorname{dist}\left(T^{* *} x^{* *}, Y\right):\left\|x^{* *}\right\| \leq 1\right\} .
$$

Proof. We write $|R(T)|=\sup \left\{\operatorname{dist}\left(T^{* *} x^{* *}, Y\right):\left\|x^{* *}\right\| \leq 1\right\}$. Clearly, $|R(T)| \leq\|R(T)\|$. Take $x^{* *} \in X^{* *}$ such that $\operatorname{dist}\left(x^{* *}, X\right) \leq 1$. For each $\varepsilon>0$ there exists $x \in X$ such that $\left\|x^{* *}-x\right\| \leq 1+\varepsilon$. Then $\operatorname{dist}\left(T^{* *} x^{* *}, Y\right)=$ $\operatorname{dist}\left(T^{* *}\left(x^{* *}-x\right), Y\right) \leq(1+\varepsilon)|R(T)|$. It follows that $\|R(T)\| \leq|R(T)|$.

Theorem 3.2. Let $X$ and $Y$ be Banach spaces and $T \in L(X, Y)$. Then

$$
\frac{1}{2} \Gamma(T) \leq \bar{\Gamma}(T) \leq\|R(T)\| \leq \Gamma(T) .
$$

Proof. Theorem 2.4 gives the first inequality. Let $y^{* *} \in Y^{* *}$ be a $w^{*}$-cluster point of a sequence in $T\left(\mathrm{~B}_{X}\right)$. Since $\overline{\mathrm{B}}_{X^{* *}}$ is $w^{*}$-compact and $T^{* *}$ 
is $w^{*}$-continuous, the set $T^{* *}\left(\overline{\mathrm{B}}_{X^{* *}}\right)$ is $w^{*}$-closed in $Y^{* *}$. Hence $y^{* *}=T^{* *} x^{* *}$ for some $x^{* *} \in \overline{\mathrm{B}}_{X^{* *}}$. Consequently, $\operatorname{dist}\left(y^{* *}, Y\right) \leq\|R(T)\|$. This shows that $\bar{\gamma}\left(T\left(\mathrm{~B}_{X}\right)\right) \leq\|R(T)\|$, which gives the second inequality of the assertion.

To prove the last inequality we modify a reasoning given in [22]. Namely, we take $x^{* *} \in \overline{\mathrm{B}}_{X^{* *}}$ and write $\vartheta=\operatorname{dist}\left(T^{* *} x^{* *}, Y\right)$. By a separation theorem there exists $y^{* * *} \in Y^{* * *}$ such that $\left\|y^{* * *}\right\|=1, y^{* * *} \mid Y=0$ and $y^{* * *}\left(T^{* *}\left(x^{* *}\right)\right)=\vartheta$.

We now fix $\varepsilon>0$ and apply the principle of local reflexivity (see $\left[18\right.$, p. 33]) to get $y_{1}^{*} \in Y^{*}$ such that $\left\|y_{1}^{*}\right\| \leq 1+\varepsilon, x^{* *}\left(T^{*} y_{1}^{*}\right)=\vartheta$, and $x_{1} \in \overline{\mathrm{B}}_{X}$ such that $\left|\left(T^{*} y_{1}^{*}\right)\left(x_{1}\right)-\vartheta\right| \leq \varepsilon$.

We next proceed by induction. Having $x_{1}, \ldots, x_{n-1} \in \overline{\mathrm{B}}_{X}$ and $y_{1}^{*}, \ldots$ $\ldots, y_{n-1}^{*} \in Y^{*}$ we choose $y_{n}^{*} \in Y^{*}$ such that $\left\|y_{n}^{*}\right\| \leq 1+\varepsilon, x^{* *}\left(T^{*} y_{n}^{*}\right)=\vartheta$ and $y_{n}^{*}\left(T x_{k}\right)=y^{* * *}\left(T^{* *} x_{k}\right)=0$ for every $k<n$. Then we find $x_{n} \in \overline{\mathrm{B}}_{X}$ such that $\left|x^{* *}\left(T^{*} y_{k}^{*}\right)-y_{k}^{*}\left(T x_{n}\right)\right| \leq \varepsilon$ for every $k \leq n$. Consequently, $y_{k}^{*}\left(T x_{n}\right) \geq$ $\vartheta-\varepsilon$ if $k \leq n$ and $y_{k}^{*}\left(T x_{n}\right)=0$ if $k>n$. It follows that $\vartheta-\varepsilon \leq \operatorname{csep}\left(T x_{n}\right)$, which in view of Lemma 3.1 finally yields $\|R(T)\| \leq \Gamma(T)$.

In [21, Prop. 1.3], $\|R(\cdot)\|$ was used to obtain a quantitative version of Gantmacher's duality theorem for weakly compact operators. This result together with Theorem 3.2 shows that similar results hold for $\Gamma$ and $\bar{\Gamma}$ as well.

The next result will play a key role in the proof of our result on the behaviour of $\Gamma$ under Calderón's complex interpolation. Let $X$ and $Y$ be Banach spaces. By $C_{X}^{\mathrm{b}}$ we denote the Banach space of all continuous functions $x: \mathbb{R} \rightarrow X$ such that $\|x\|=\sup \{\|x(t)\|: t \in \mathbb{R}\}<\infty$. Let $(\mathbb{R}, \Sigma, \nu)$ be a measure space, where the measure $\nu$ is absolutely continuous with respect to the Lebesgue measure $\mu$ on $\mathbb{R}$ and $\nu(\mathbb{R})=1$. The Banach space of all (equivalence classes of) $\nu$-measurable functions $y: \mathbb{R} \rightarrow Y$ such that $\|y\|=\int_{\mathbb{R}}\|y(t)\| d \nu(t)<\infty$ will be denoted by $L_{Y}^{1}(\nu)$. Next, $\Lambda_{Y^{*}}$ denotes the space of all functions $f: \mathbb{R} \rightarrow Y^{*}$ such that the function $t \mapsto\langle y, f(t)\rangle$ is $\nu$-integrable for every $y \in Y$ (here $\langle y, f(t)\rangle=f(t)(y)$ ). For a detailed characterization of $\left(L_{Y}^{1}(\nu)\right)^{*}$ in terms of $\Lambda_{Y^{*}}$ we refer to $[20]$.

Theorem 3.3. Let $X, Y$ be Banach spaces and $T \in L(X, Y)$. Let $\widehat{T} \in$ $L\left(C_{X}^{\mathrm{b}}, L_{Y}^{1}(\nu)\right)$ be given by the formula $(\widehat{T} f)(t)=T(f(t))$ for every $f \in C_{X}^{\mathrm{b}}$. Then $\Gamma(\widehat{T})=\Gamma(T)$.

Proof. Considering the subspace of all constant functions in $C_{X}^{\mathrm{b}}$ one can easily see that $\Gamma(\widehat{T}) \geq \Gamma(T)$. To prove that $\Gamma(\widehat{T}) \leq \Gamma(T)$ we use formula (2.1). We fix $\varepsilon>0$ and choose sequences $\left(y_{n}\right) \subset T\left(\mathrm{~B}_{C_{X}^{\mathrm{b}}}\right)$ and 
$\left(F_{n}\right) \subset\left(L_{Y}^{1}(\nu)\right)^{*}$ with $\left\|F_{n}\right\| \leq 1$ for every $n$ such that $\Gamma(\widehat{T})-\varepsilon \leq \vartheta_{1}-\vartheta_{2}$ where $\vartheta_{1}=\lim _{n} \lim _{k} F_{n}\left(y_{k}\right)$ and $\vartheta_{2}=\lim _{k} \lim _{n} F_{n}\left(y_{k}\right)$. Since $L^{1}(\nu)$ is separable, for each $n$ there exists $f_{n} \in \Lambda_{Y^{*}}$ such that $\left\|f_{n}(t)\right\| \leq 1$ for all $t \in \mathbb{R}$ and

$$
F_{n}(y)=\int_{\mathbb{R}}\left\langle y(t), f_{n}(t)\right\rangle d \nu(t)
$$

for every $y \in L_{Y}^{1}(\nu)$ (see [20]). Let $\left\langle y_{k}, f_{n}\right\rangle$ denote the function $t \mapsto$ $\left\langle y_{k}(t), f_{n}(t)\right\rangle$. Obviously, the functions $\left\langle y_{k}, f_{n}\right\rangle, k, n \geq 1$, are equiintegrable, so they form a relatively weakly compact set in $L^{1}(\nu)$ (see [17, p. 93]). By the Eberlein-Shmul'yan and Mazur theorems (see [17, pp. 11, 18]), for each sequence in a relatively weakly compact set of a Banach space there exists a convergent sequence of its scc. Next, each convergent sequence in $L^{1}(\nu)$ has a subsequence which converges $\nu$-a.e. to the same limit (see $[19$, p. 150]). Therefore we can find a sequence $\left(y_{k}^{1}\right)$ of scc for $\left(y_{k}\right)$ such that $\left\langle y_{k}^{1}, f_{1}\right\rangle$ tends $\nu$-a.e. to some $g_{1} \in L^{1}(\nu)$.

We now proceed by induction. For $n \geq 2$ choose a sequence $\left(y_{k}^{n}\right)$ of scc for $\left(y_{k}^{n-1}\right)$ such that $\left\langle y_{k}^{n}, f_{n}\right\rangle$ tends $\nu$-a.e. to $g_{n} \in L^{1}(\nu)$ as $k \rightarrow \infty$. Write $y_{k}^{\prime}=y_{k}^{k}$ for every $k$. Then $\left(y_{k}^{\prime}\right)_{k \geq m}$ is a sequence of scc for $\left(y_{k}^{m}\right)$ and $\left\langle y_{k}^{\prime}, f_{n}\right\rangle$ also tends $\nu$-a.e. to $g_{n}$ as $k \rightarrow \infty$ for every $n$. We next choose a sequence $\left(g_{n}^{\prime}\right)$ of scc for $\left(g_{n}\right)$ convergent $\nu$-a.e. to some $g \in L^{1}(\nu)$. Then $g_{n}^{\prime}=\sum_{i=m_{n}+1}^{m_{n+1}} \lambda_{i}^{(n)} g_{i}$, where $\left(m_{n}\right)$ is an increasing sequence of positive integers, $\lambda_{i}^{(n)} \geq 0$ and $\sum_{i=m_{n}+1}^{m_{n+1}} \lambda_{i}^{(n)}=1$. We set

$$
f_{n}^{\prime}=\sum_{i=m_{n}+1}^{m_{n+1}} \lambda_{i}^{(n)} f_{i} \text { and } F_{n}^{\prime}=\sum_{i=m_{n}+1}^{m_{n+1}} \lambda_{i}^{(n)} F_{i} .
$$

In this way we have obtained the sequences $\left(y_{k}^{\prime}\right) \subset T\left(\mathrm{~B}_{C_{X}^{\mathrm{b}}}\right),\left(f_{n}^{\prime}\right) \subset \Lambda_{Y^{*}}$ and $\left(F_{n}^{\prime}\right) \subset\left(L_{Y}^{1}(\nu)\right)^{*}$ with the following properties: $\left\|F_{n}^{\prime}\right\| \leq 1, \lim _{n} \lim _{k} F_{n}^{\prime}\left(y_{k}^{\prime}\right)$ $=\vartheta_{1}, \lim _{k} \lim _{n} F_{n}^{\prime}\left(y_{k}^{\prime}\right)=\vartheta_{2}$,

$$
F_{n}^{\prime}(y)=\int_{\mathbb{R}}\left\langle y(t), f_{n}^{\prime}(t)\right\rangle d \nu(t)
$$

for all $y \in L_{Y}^{1}(\nu), n \geq 1$, and $\lim _{n} \lim _{k}\left\langle y_{k}^{\prime}, f_{n}^{\prime}\right\rangle=g, \nu$-a.e.

Similarly, we can choose a sequence $\left(f_{n}^{\prime \prime}\right)$ of scc for $\left(f_{n}^{\prime}\right)$ such that $\left\langle y_{k}^{\prime}, f_{n}^{\prime \prime}\right\rangle$ tends $\nu$-a.e. to some $h_{k} \in L^{1}(\nu)$ for each $k$. Next, we take a sequence $\left(y_{k}^{\prime \prime}\right)$ of scc for $\left(y_{k}^{\prime}\right)$ and the corresponding sequence $\left(F_{n}^{\prime \prime}\right)$ of scc for $\left(F_{n}^{\prime}\right)$ such that the following limit exists:

$$
\lim _{k} \lim _{n}\left\langle y_{k}^{\prime \prime}, f_{n}^{\prime \prime}\right\rangle=h, \quad \nu \text {-a.e. }
$$

Then $\lim _{n} \lim _{k}\left\langle y_{k}^{\prime \prime}, f_{n}^{\prime \prime}\right\rangle=g, \nu$-a.e. 
The Lebesgue dominated convergence theorem yields

$$
\begin{aligned}
\int_{\mathbb{R}}(g(t)- & h(t)) d \nu(t) \\
= & \lim _{n} \lim _{k} \int_{\mathbb{R}}\left\langle y_{k}^{\prime \prime}(t), f_{n}^{\prime \prime}(t)\right\rangle d \nu(t)-\lim _{k} \lim _{n} \int_{\mathbb{R}}\left\langle y_{k}^{\prime \prime}(t), f_{n}^{\prime \prime}(t)\right\rangle d \nu(t) \\
= & \lim _{n} \lim _{k} F_{n}^{\prime \prime}\left(y_{k}^{\prime \prime}\right)-\lim _{k} \lim _{n} F_{n}^{\prime \prime}\left(y_{k}^{\prime \prime}\right)=\vartheta_{1}-\vartheta_{2} \geq \Gamma(\widehat{T})-\varepsilon .
\end{aligned}
$$

Since $\nu(\mathbb{R})=1$, there exists $t_{0}$ such that

$$
\lim _{n} \lim _{k}\left\langle y_{k}^{\prime \prime}\left(t_{0}\right), f_{n}^{\prime \prime}\left(t_{0}\right)\right\rangle-\lim _{k} \lim _{n}\left\langle y_{k}^{\prime \prime}\left(t_{0}\right), f_{n}^{\prime \prime}\left(t_{0}\right)\right\rangle \geq \Gamma(\widehat{T})-\varepsilon,
$$

which gives $\Gamma(T) \geq \Gamma(\widehat{T})-\varepsilon$. By the arbitrary choice of $\varepsilon>0$ we conclude that $\Gamma(T) \geq \Gamma(\widehat{T})$.

4. Complex interpolation and the measure $\Gamma$. Complex Banach spaces $A_{0}$ and $A_{1}$ are compatible if they are continuously embedded in a common Hausdorff complex topological vector space. Then $A_{0} \cap A_{1}, A_{0}+A_{1}$ with the norms

$\|a\|_{A_{0} \cap A_{1}}=\max \left\{\|a\|_{A_{0}},\|a\|_{A_{1}}\right\}, \quad\|a\|_{A_{0}+A_{1}}=\inf _{a=a_{0}+a_{1}}\left\{\left\|a_{0}\right\|_{A_{0}}+\left\|a_{1}\right\|_{A_{1}}\right\}$

are Banach spaces as well. Let $\bar{A}=\left(A_{0}, A_{1}\right)$ denote a pair of compatible Banach spaces $A_{0}$ and $A_{1}$. A Banach space $A$ is said to be an intermediate space with respect to $\bar{A}$ if

$$
A_{0} \cap A_{1} \subset A \subset A_{0}+A_{1}
$$

and both inclusions are continuous. Let $\bar{A}=\left(A_{0}, A_{1}\right)$ and $\bar{B}=\left(B_{0}, B_{1}\right)$ be two pairs of compatible Banach spaces and let $T$ be a linear operator from $A_{0}+A_{1}$ into $B_{0}+B_{1}$. We write $T: \bar{A} \rightarrow \bar{B}$ if $T \in L\left(A_{0}, B_{0}\right)$ and $T \in L\left(A_{1}, B_{1}\right)$, viewed as the restrictions of $T$. If $A$ and $B$ are intermediate spaces with respect to $\bar{A}$ and $\bar{B}$ respectively, and $T: \bar{A} \rightarrow \bar{B}$ implies that $T \in L(A, B)$, then $A$ and $B$ are said to be interpolation spaces with respect to $\bar{A}$ and $\bar{B}$.

We recall Calderón's construction of interpolation spaces, i.e. the socalled complex interpolation method (see [10]). Given a pair $\bar{A}=\left(A_{0}, A_{1}\right)$ of compatible spaces we denote by $\mathcal{F}(\bar{A})$ the space of all functions $f$ on the strip $S=\{z: 0 \leq \operatorname{Re} z \leq 1\}$ with values in $A_{0}+A_{1}$ which are bounded and continuous on $S$ and analytic on its interior, and moreover the functions $t \mapsto f(j+i t), j=0,1$, from $\mathbb{R}$ into $A_{j}$ are continuous and tend to zero as $|t| \rightarrow \infty$. The space $\mathcal{F}(\bar{A})$ becomes a Banach space if we provide it with the norm

$$
\|f\|_{\mathcal{F}}=\max \left\{\sup _{t}\|f(i t)\|_{A_{0}}, \sup _{t}\|f(1+i t)\|_{A_{1}}\right\} .
$$


Given $0<\theta<1$ we consider the space

$$
A_{[\theta]}=\left\{a \in A_{0}+A_{1}: a=f(\theta), f \in \mathcal{F}(\bar{A})\right\}
$$

with the norm of $a \in A_{[\theta]}$ given by

$$
\|a\|_{[\theta]}=\inf \left\{\|f\|_{\mathcal{F}}: f(\theta)=a, f \in \mathcal{F}(\bar{A})\right\} .
$$

It is an intermediate space with respect to $\bar{A}$. Moreover, if $\bar{A}=\left(A_{0}, A_{1}\right)$ and $\bar{B}=\left(B_{0}, B_{1}\right)$ are pairs of compatible spaces and $T: \bar{A} \rightarrow \bar{B}$ then

$$
\|T\|_{[\theta]} \leq\|T\|_{0}^{1-\theta}\|T\|_{1}^{\theta},
$$

where $\|T\|_{[\theta]}$ and $\|T\|_{j}, j=0,1$, are the norms of the operators $T: A_{[\theta]} \rightarrow$ $B_{[\theta]}$ and $T: A_{j} \rightarrow B_{j}, j=0,1$, respectively. This shows in particular that $A_{[\theta]}$ and $B_{[\theta]}$ are interpolation spaces with respect to $\bar{A}$ and $\bar{B}$.

We shall prove an analogous inequality for the measure of weak noncompactness $\Gamma$ for operators. Let $P_{j}, j=0,1$, be the Poisson kernels for the strip $S$. Then $\int_{\mathbb{R}} P_{0}(\theta, t) d \mu(t)=1-\theta$ and $\int_{\mathbb{R}} P_{1}(\theta, t) d \mu(t)=\theta$. Therefore, the formulae

$$
\nu_{0}(K)=\frac{1}{1-\theta} \int_{K} P_{0}(\theta, t) d \mu(t), \quad \nu_{1}(K)=\frac{1}{\theta} \int_{K} P_{1}(\theta, t) d \mu(t),
$$

for each $\mu$-measurable set $K$ in $\mathbb{R}$, define probability measures $\nu_{0}$ and $\nu_{1}$ which are absolutely continuous with respect to the Lebesgue measure $\mu$. We put $X_{j}=L_{A_{j}}^{1}\left(\nu_{j}\right), j=0,1$. Given $f \in \mathcal{F}(\bar{A})$ we denote by $f_{j}$ the function $t \mapsto f(j+i t)$ for $j=0,1$. Then

$$
\|a\|_{[\theta]} \leq\left\|f_{0}\right\|_{X_{0}}^{1-\theta}\left\|f_{1}\right\|_{X_{1}}^{\theta}
$$

whenever $f(\theta)=a \in A_{[\theta]}$ and $f \in \mathcal{F}(\bar{A})$ (see $[10]$ ).

Theorem 4.1. Let $\bar{A}=\left(A_{0}, A_{1}\right)$ and $\bar{B}=\left(B_{0}, B_{1}\right)$ be pairs of compatible Banach spaces, $0<\theta<1$ and $T: \bar{A} \rightarrow \bar{B}$. Then

$$
\Gamma_{[\theta]}(T) \leq \Gamma_{0}(T)^{1-\theta} \Gamma_{1}(T)^{\theta},
$$

where $\Gamma_{[\theta]}(T)$ and $\Gamma_{j}(T), j=0,1$, are the measures of weak noncompactness $\Gamma$ for the operators $T: A_{[\theta]} \rightarrow B_{[\theta]}$ and $T: A_{j} \rightarrow B_{j}, j=0,1$, respectively.

Proof. We fix $\varepsilon>0$ and a sequence $\left(b_{n}\right) \subset T\left(\mathrm{~B}_{A_{[\theta]}}\right)$. For each $n$ there exist $a_{n} \in \mathrm{B}_{A_{[\theta]}}$ with $b_{n}=T a_{n}$ and a function $f_{n} \in \mathcal{F}(\bar{A})$ such that $\left\|f_{n}\right\|_{\mathcal{F}}<1$ and $f_{n}(\theta)=a_{n}$. Then $T \circ f_{n}=g_{n} \in \mathcal{F}(\bar{B})$ and $g_{n}(\theta)=b_{n}$. Let $g_{j, n}$ denote the function $t \mapsto g_{n}(j+i t)$ and let $Y_{j}=L_{B_{j}}^{1}\left(\nu_{j}\right)$ for $j=0,1$. Of course, $g_{j, n} \in Y_{j}$. By Theorem 2.1 we can find a sequence $\left(g_{0, n}^{\prime}\right)$ of scc for $\left(g_{0, n}\right)$ such that $\left\|h_{1}-h_{2}\right\|_{Y_{0}} \leq \operatorname{csep}\left(g_{0, n}^{\prime}\right)+\varepsilon$ for every pair $h_{1}, h_{2}$ of scc for $\left(g_{0, n}^{\prime}\right)$. Then $g_{0, n}^{\prime}=\sum_{i=m_{n}+1}^{m_{n+1}} \alpha_{i}^{(n)} g_{0, i}$, where $\left(m_{n}\right)$ is an increasing sequence of positive integers, the numbers $\alpha_{i}^{(n)}$ are nonnegative and $\sum_{i=m_{n}+1}^{m_{n+1}} \alpha_{i}^{(n)}=1$. 
Let $g_{1, n}^{\prime}=\sum_{i=m_{n}+1}^{m_{n+1}} \alpha_{i}^{(n)} g_{1, i}$. Applying once again Theorem 2.1 we choose a sequence $\left(g_{1, n}^{\prime \prime}\right)$ of scc for $\left(g_{1, n}^{\prime}\right)$ such that $\left\|g_{1, m}^{\prime \prime}-g_{1, k}^{\prime \prime}\right\|_{Y_{1}} \leq \operatorname{csep}\left(g_{1, n}^{\prime \prime}\right)$ $+\varepsilon$ for all $m, k$. Similarly, $g_{1, n}^{\prime \prime}=\sum_{i=l_{n}+1}^{l_{n+1}} \beta_{i}^{(n)} g_{1, i}^{\prime}$, where $\left(l_{n}\right)$ and $\beta_{i}^{(n)}$ satisfy the same conditions as $\left(m_{n}\right)$ and $\alpha_{i}^{(n)}$. We set $g_{0, n}^{\prime \prime}=\sum_{i=l_{n}+1}^{l_{n+1}} \beta_{i}^{(n)} g_{0, i}^{\prime}$. In this way we have obtained the sequences $\left(g_{j, n}^{\prime \prime}\right), j=0,1$, for which

$$
\left\|g_{j, m}^{\prime \prime}-g_{j, k}^{\prime \prime}\right\|_{Y_{j}} \leq \operatorname{csep}\left(g_{j, n}^{\prime \prime}\right)+\varepsilon \text {. }
$$

Let $g_{n}^{\prime \prime}=\sum_{k=l_{n}+1}^{l_{n+1}} \beta_{k}^{(n)}\left(\sum_{i=m_{k}+1}^{m_{k+1}} \alpha_{i}^{(k)} g_{i}\right)$ and $\widehat{T}_{j}: C_{A_{j}}^{\mathrm{b}} \rightarrow Y_{j}, j=0,1$, be the operator derived from $T$ as in Theorem 3.3. Then

$$
\begin{aligned}
\operatorname{csep}\left(b_{n}\right) & \leq \operatorname{csep}\left(g_{n}^{\prime \prime}(\theta)\right) \leq\left\|g_{1}^{\prime \prime}(\theta)-g_{2}^{\prime \prime}(\theta)\right\|_{[\theta]} \\
& \leq\left\|g_{0,1}^{\prime \prime}-g_{0,2}^{\prime \prime}\right\|_{Y_{0}}^{1-\theta}\left\|g_{1,1}^{\prime \prime}-g_{1,2}^{\prime \prime}\right\|_{Y_{1}}^{\theta} \\
& \leq\left(\operatorname{csep}\left(g_{0, n}^{\prime \prime}\right)+\varepsilon\right)^{1-\theta}\left(\operatorname{csep}\left(g_{1, n}^{\prime \prime}\right)+\varepsilon\right)^{\theta} \\
& \leq\left(\Gamma\left(\widehat{T}_{0}\right)+\varepsilon\right)^{1-\theta}\left(\Gamma\left(\widehat{T}_{1}\right)+\varepsilon\right)^{\theta},
\end{aligned}
$$

where the third inequality is a consequence of (4.1). Theorem 3.3 and the arbitrary choice of $\left(b_{n}\right)$ and $\varepsilon>0$ yield the assertion of our theorem.

Combining the above result with Theorem 3.2 we obtain the following corollary.

COROllary 4.2. Under the assumptions of Theorem 4.1,

$$
\|R(T)\|_{[\theta]} \leq 2\|R(T)\|_{0}^{1-\theta}\|R(T)\|_{1}^{\theta},
$$

where $\|R(T)\|_{[\theta]}$ and $\|R(T)\|_{j}, j=0,1$, are the norms of the operators $R\left(T \mid A_{[\theta]}\right)$ and $R\left(T \mid A_{j}\right), j=0,1$, respectively.

An analogous result holds for the measure $\bar{\Gamma}$. Theorem 4.1 also gives the next corollary.

Corollary 4.3. If an operator $T: A_{0} \rightarrow B_{0}$ or $T: A_{1} \rightarrow B_{1}$ is weakly compact, then so is $T: A_{[\theta]} \rightarrow B_{[\theta]}$ for all $0<\theta<1$.

\section{References}

[1] R. R. Akhmerov, M. I. Kamenskiǔ, A. S. Potapov, A. E. Rodkina and B. N. Sadovskiı̌, Measures of Noncompactness and Condensing Operators, Birkhäuser, Basel, 1992.

[2] A. G. Aksoy and L. Maligranda, Real interpolation and measure of weak noncompactness, Math. Nachr. 175 (1995), 5-12.

[3] J. Appell and E. De Pascale, Su alcuni parametri connessi con la misura di non compattezza di Hausdorff in spazi di funzioni misurabili, Boll. Un. Mat. Ital. B 3 (1984), 497-515.

[4] K. Astala and H.-O. Tylli, Seminorms related to weak compactness and to Tauberian operators, Math. Proc. Cambridge Philos. Soc. 107 (1990), 367-375. 
[5] J. M. Ayerbe Toledano, T. Domínguez Benavides and G. López Acedo, Measures of Noncompactness in Metric Fixed Point Theory, Birkhäuser, Basel, 1997.

[6] J. Banaś, Applications of measure of weak noncompactness and some classes of operators in the theory of functional equations in the Lebesgue space, Proc. of the Second World Congress of Nonlinear Analysts, Part 6 (Athens, 1996), Nonlinear Anal. 30 (1997), 3283-3293.

[7] J. Banaś and A. Martinón, Measures of weak noncompactness in Banach sequence spaces, Portugal. Math. 52 (1995), 131-138.

[8] J. Banaś and J. Rivero, On measures of weak noncompactness, Ann. Mat. Pura Appl. 151 (1988), 213-224.

[9] B. Beauzamy, Espaces d'interpolation réels: topologie et géométrie, Lecture Notes in Math. 666, Springer, Berlin, 1978.

[10] A. P. Calderón, Intermediate spaces and interpolation, the complex method, Studia Math. 24 (1964), 113-190.

[11] F. Cobos, Interpolation theory and measures related to operator ideals, in: Nonlinear Analysis, Function Spaces and Applications, Vol. 6, M. Krbec and A. Kufner (eds.), Olympia Press, Prague, 1999, 93-118.

[12] F. Cobos, P. Fernández-Martínez and A. Martínez, Interpolation of the measure of non-compactness by the real method, Studia Math. 135 (1999), 25-38.

[13] F. Cobos, A. Manzano and A. Martínez, Interpolation theory and measures related to operator ideals, Quart. J. Math. Oxford Ser. (2) 50 (1999), 401-416.

[14] F. Cobos and A. Martínez, Extreme estimates for interpolated operators by the real method, J. London Math. Soc. (2) 60 (1999), 860-870.

[15] - - - Remarks on interpolation properties of the measure of weak non-compactness and ideal variations, Math. Nachr. 208 (1999), 93-100.

[16] F. S. De Blasi, On a property of the unit sphere in a Banach space, Bull. Math. Soc. Sci. Math. R. S. Roumanie 21 (69) (1977), 259-262.

[17] J. Diestel, Sequences and Series in Banach Spaces, Springer, New York, 1984.

[18] D. van Dulst, Reflexive and Superreflexive Banach Spaces, Math. Centrum, Amsterdam, 1978.

[19] N. Dunford and J. T. Schwartz, Linear Operators. Part I: General Theory, Interscience, New York, 1958.

[20] R. Edwards, Functional Analysis. Theory and Applications, Holt, Rinehart and Winston, New York, 1965.

[21] M. González, E. Saksman and H.-O. Tylli, Representing non-weakly compact operators, Studia Math. 113 (1995), 265-282.

[22] R. C. James, Weak compactness and reflexivity, Israel J. Math. 2 (1964), 101-119.

[23] M. I. Kadec and A. Pełczyński, Bases, lacunary sequences and complemented subspaces in $L_{p}$, Studia Math. 21 (1962), 161-176.

[24] A. Kryczka, S. Prus and M. Szczepanik, Measure of weak noncompactness and real interpolation of operators, Bull. Austral. Math. Soc. 62 (2000), 389-401.

[25] L. Maligranda, Interpolation between sum and intersection of Banach spaces, J. Approx. Theory 47 (1986), 42-53.

[26] D. P. Milman and V. D. Milman, The geometry of imbeddings with empty intersection. The structure of the unit sphere in a non-reflexive space, Mat. Sb. 66 (1965), 109-118 (in Russian).

[27] V. D. Milman, Geometric theory of Banach spaces. II. Geometry of the unit ball, Uspekhi Mat. Nauk 26 (1971), no. 6, 73-149 (in Russian); English transl.: Russian Math. Surveys 26 (1971), 79-163. 
[28] M. F. Teixeira and D. E. Edmunds, Interpolation theory and measures of noncompactness, Math. Nachr. 104 (1981), 129-135.

[29] K.-W. Yang, Operators invertible modulo the weakly compact operators, Pacific J. Math. 71 (1977), 559-564.

Institute of Mathematics

Maria Curie-Skłodowska University

20-031 Lublin, Poland

E-mail: akryczka@golem.umcs.lublin.pl

bsprus@golem.umcs.lublin.pl

Received September 12, 2000

Revised version March 8, 2001 